\title{
Goddard Laser for Absolute Measurement of Radiance for Instrument Calibration in the Ultraviolet to Short Wave Infrared
}

Brendan McAndrew[a], Joel McCorkel[a], Timothy Shuman[b], Barbara Zukowski[c], Aboubakar Traore[d], Michael Rodriguez[e], Steven Brown[f], and John Woodward[f]

[a]NASA Goddard Space Flight Center, Greenbelt, MD, USA 20771

[b]Fibertek, Herndon, VA, USA 20171

[c]Ball Aerospace, Lanham, MD, USA 20706

[d]NASA Langley Research Center, Hampton, VA, USA 23666

[e]Sigma Space Corporation, Lanham, MD, USA 20706

[f]National Institute of Standards and Technology, Gaithersburg, MD, USA 20899 


\section{Introduction}

- Purpose

- Absolute radiometric scale

- Calibration scheme

- Narrow linewidth tunable sources
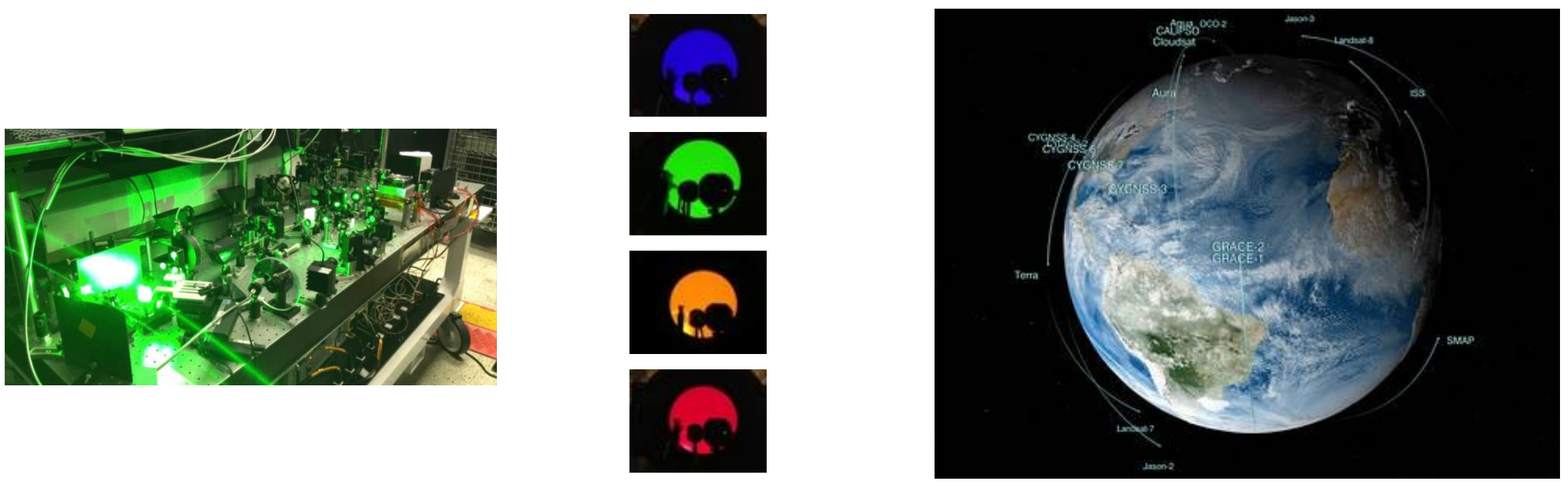

Light source

$\rightarrow$

radiometry

$\rightarrow \quad$ prelaunch calibration for instruments 


\section{Purpose}

- Narrow linewidth, high spectral bandwidth sources provide higher signal and dynamic range, improved wavelength and radiance accuracy over broadband lamp based techniques

- More straightforward measurement and data interpretation - flat field, full signal level

- Enables more science, reduced mission lifetimes \& cost

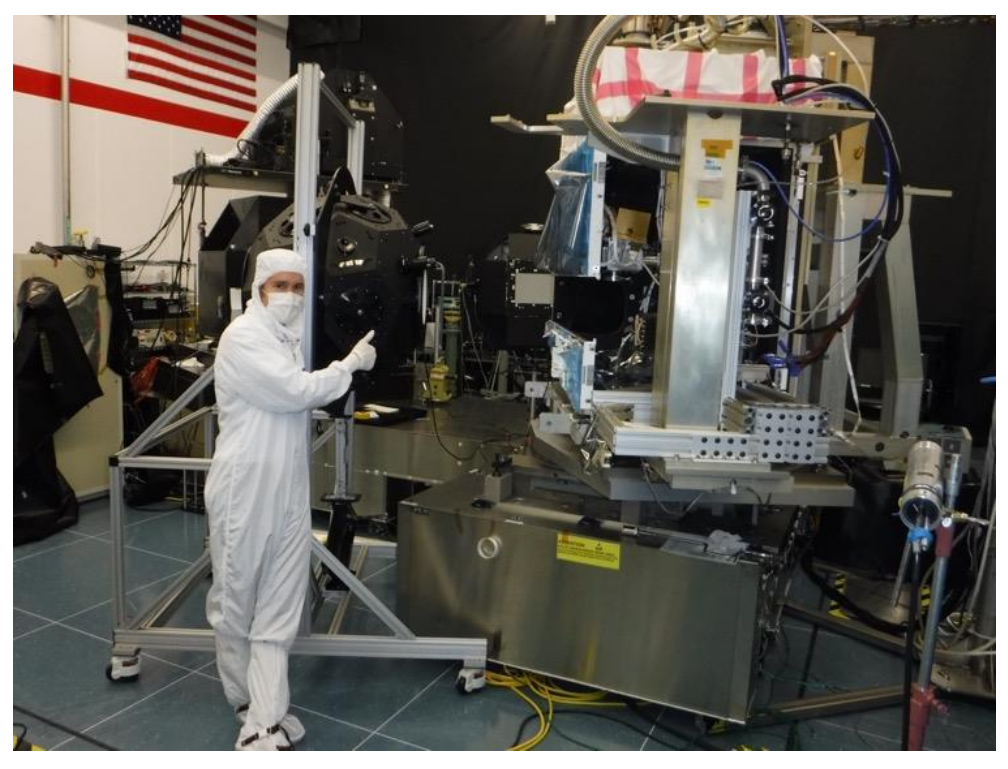




\section{Absolute radiometric scale}

Radiance: power per unit area per unit solid angle $L=\frac{P}{A * \Omega}$

Spectral radiance: radiance per unit wavelength $L_{\lambda}=L / \Delta \lambda$

Greatest uncertainty is in optical power $P$

Area and solid angle are both traceable to meters

Optical power measured with electrical substitution radiometer and traceable to electrical units of measure

L-1 DET-8 SN 107 Radiance Responsivity
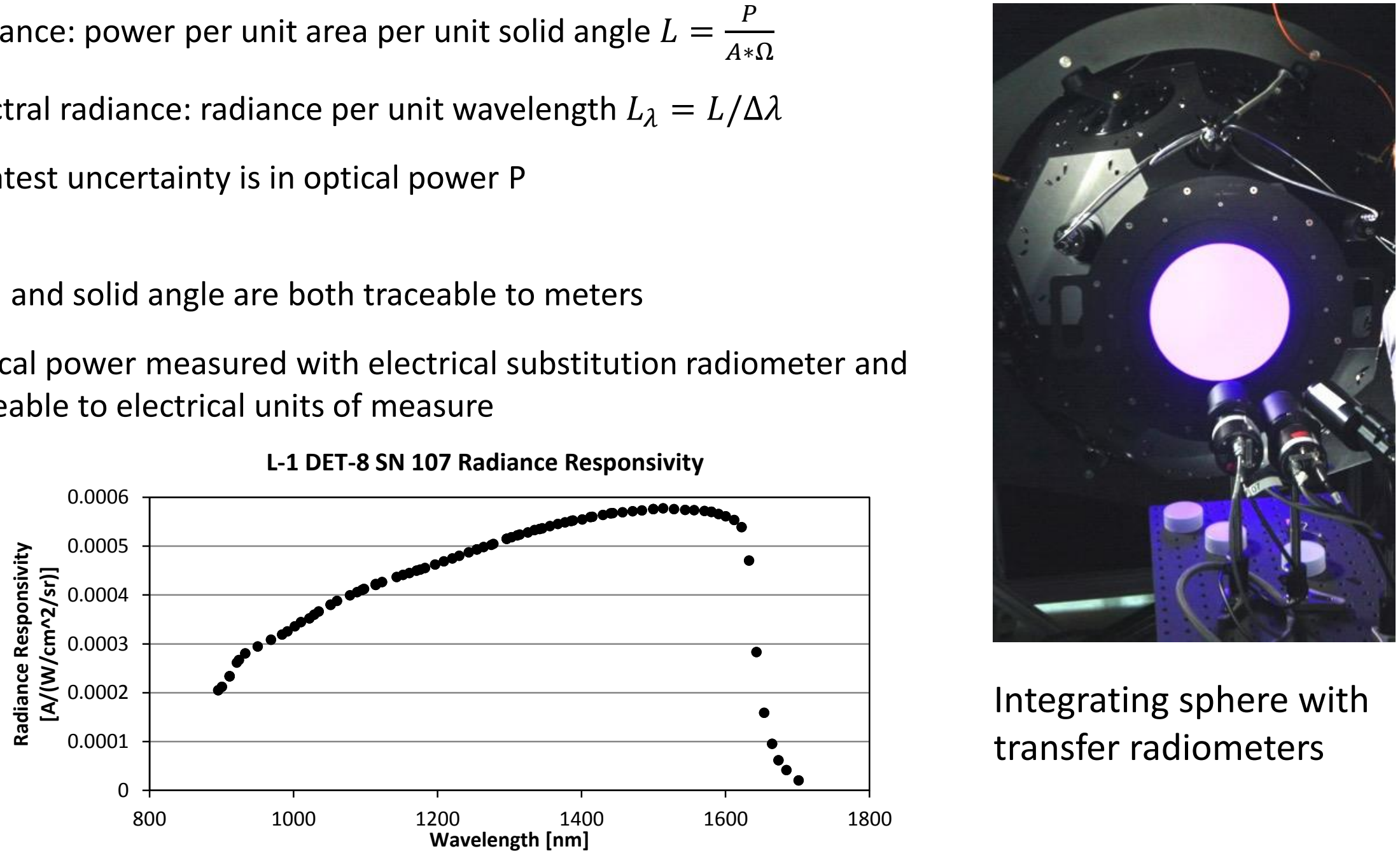

Integrating sphere with transfer radiometers

Narrow linewidth source eliminates error due to convolution of source spectrum with radiometer responsivity 


\section{Traceability path}
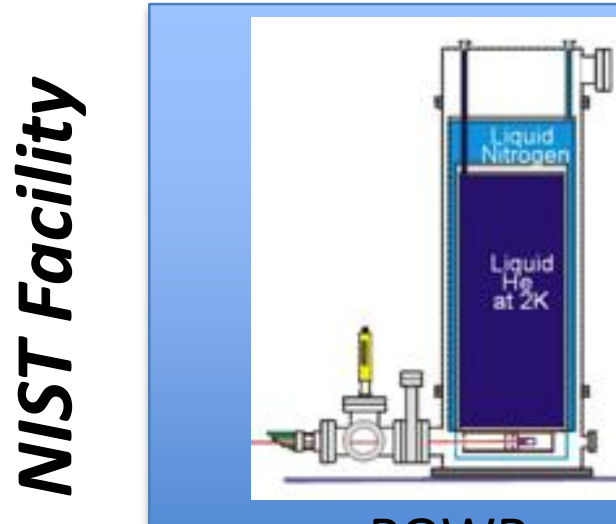

POWR

Primary Optical Watt Radiometer

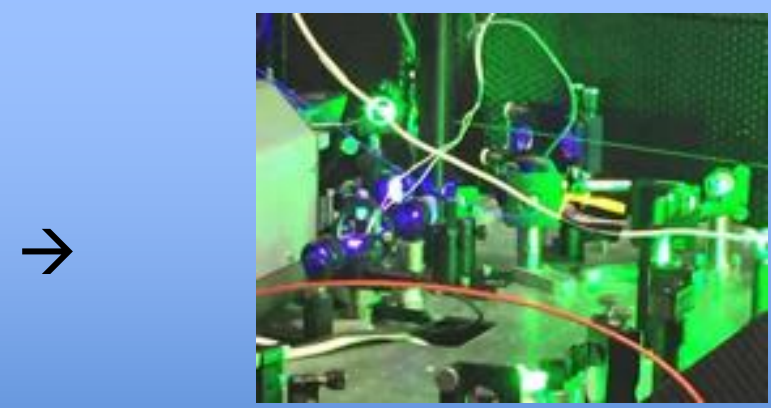

Stabilized laser source is used to transfer radiometric scale from POWR to portable transfer radiometer via another standard radiometer

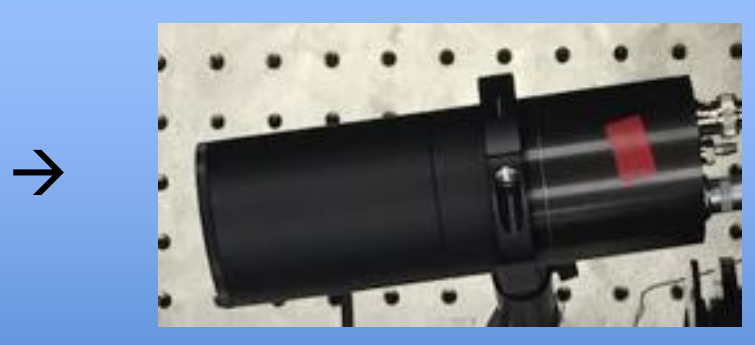

LTD-11 \#107 transfer radiometer

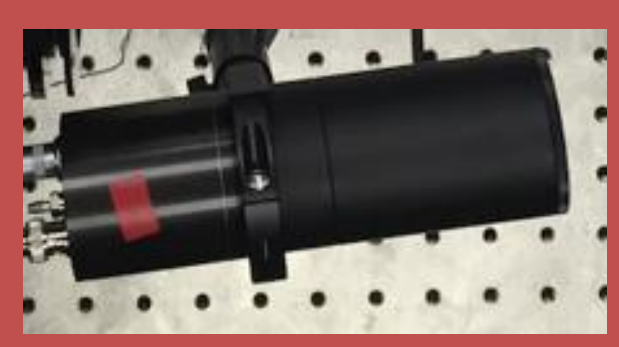

LTD-11 \#107 transfer radiometer

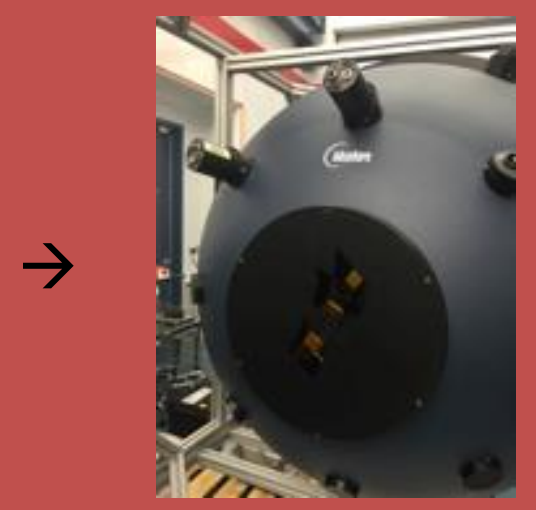

Sphere Monitor

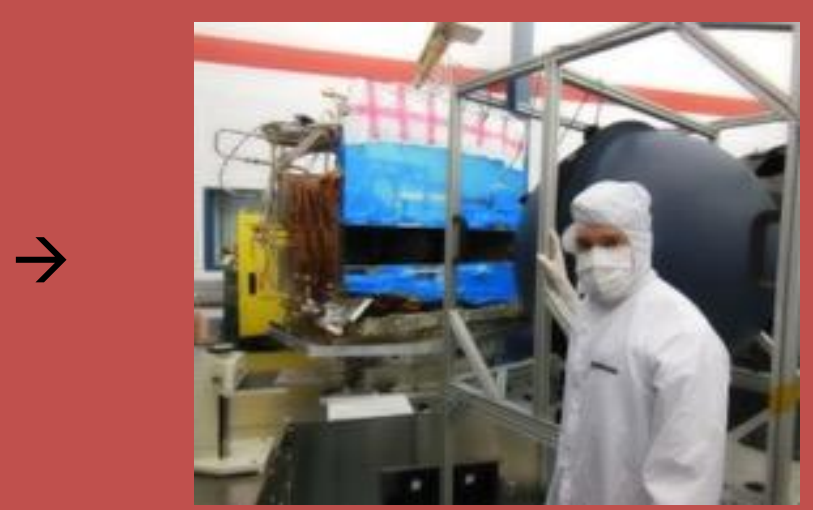

Satellite/airborne sensor 


\section{Tunable sources}

Desired properties:

- Radiometrically stable

- Wavelength range covering the solar spectrum, 300 to $2500 \mathrm{~nm}$

- Signal level comparable to maximum reflected solar radiance (snow and cloud cover at high sun angles)

- Linewidth $<$ instrument under test

- Portability, minimal setup and facility infrastructure requirements

- Time efficiency: automated wavelength tuning, synchronized tuning with shutter cycling, instrument data collects

\section{Solar spectrum}

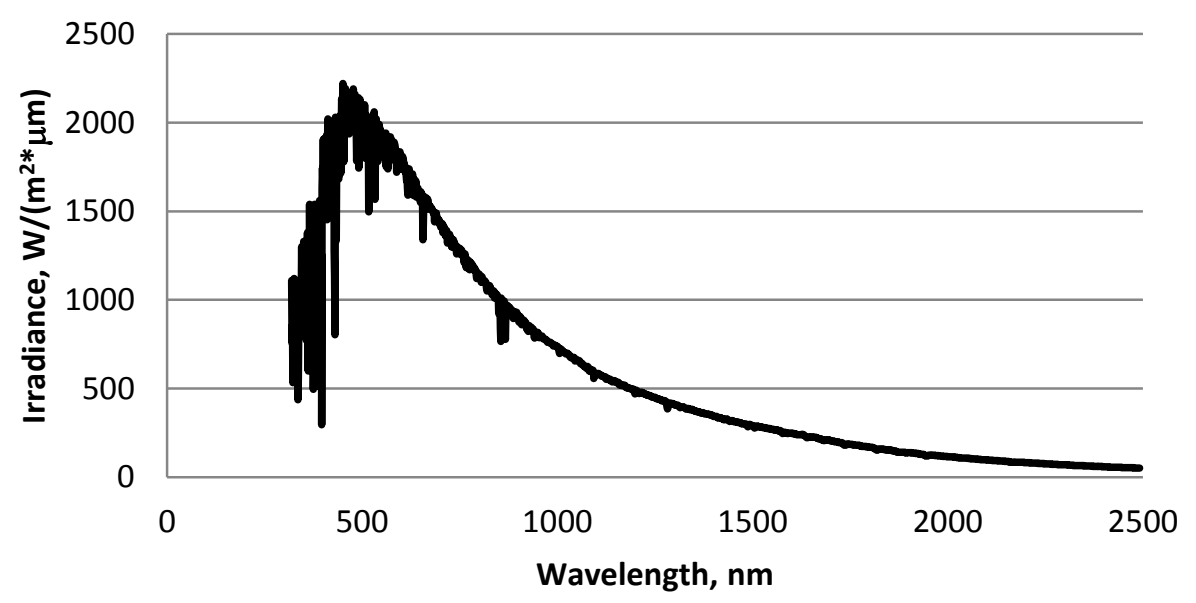

Lamp/monochromator

Titanium sapphire

Dye

Tunable diode

Optical parametric oscillator

- Reliability: critical path operations 


\section{Custom LBO OPO}

\section{NIR-OPO $680-1100 \mathrm{~nm}+1200-2200 \mathrm{~nm}$}

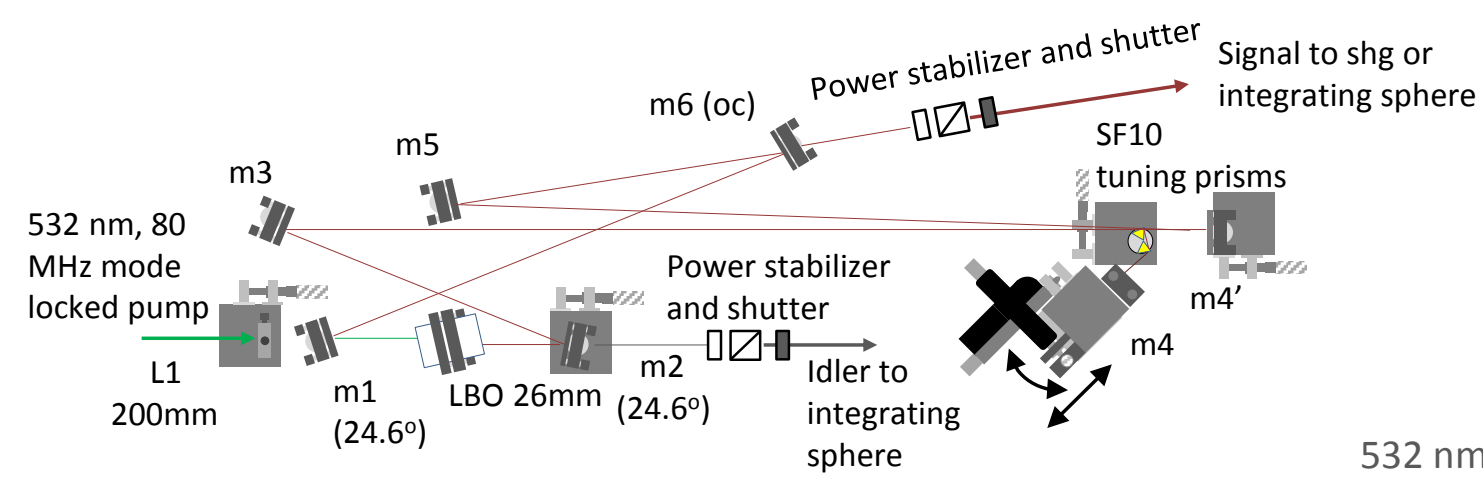

$532 \mathrm{~nm}$ pumped LBO OPO temperature tuning $(\mathrm{e} \rightarrow \mathrm{O}+\mathrm{O})$

SWIR-OPO 1080-1400 nm + 540-700 nm
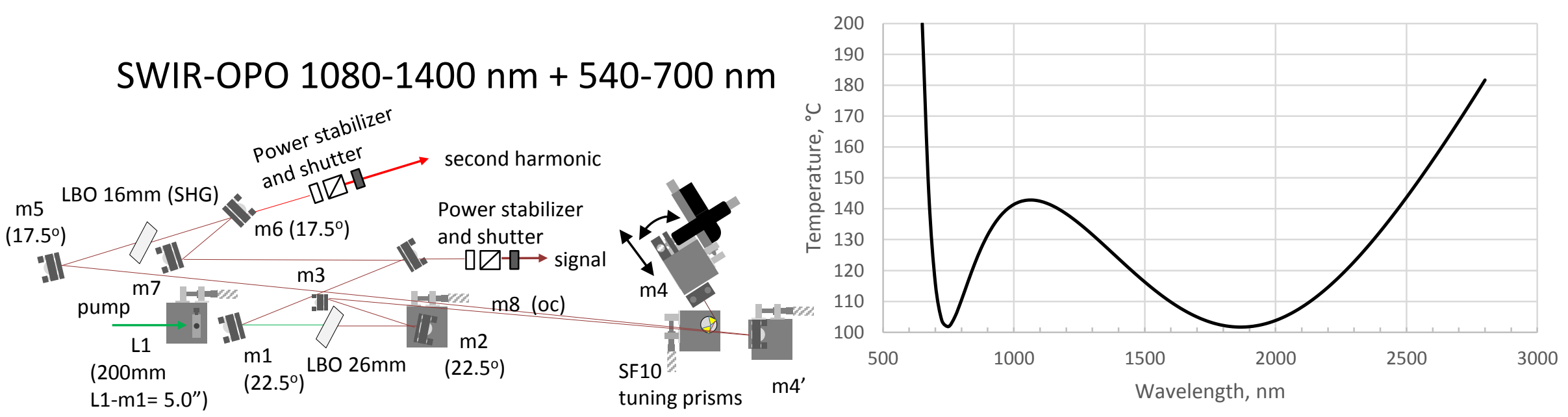


\section{Automation}

Real time display + recording of wavelength, radiance, shutter state, and OPO parameters Light and dark dwell time

Scan wavelength interval

Automated tuning via parameter look up table

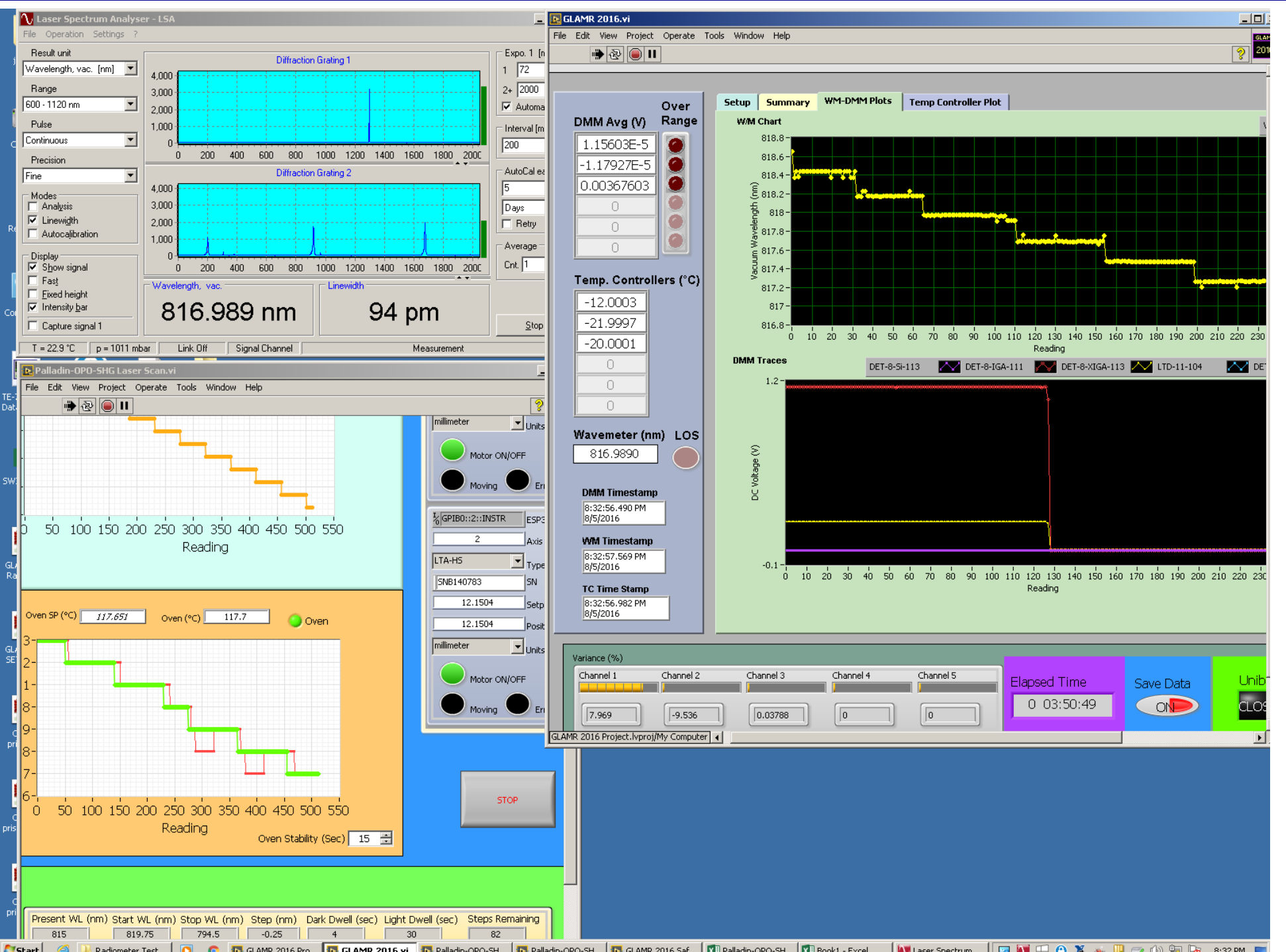

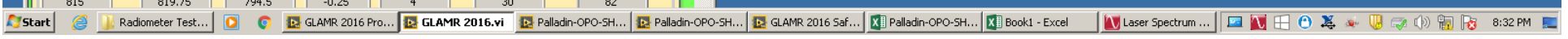




\section{Example calibration data}

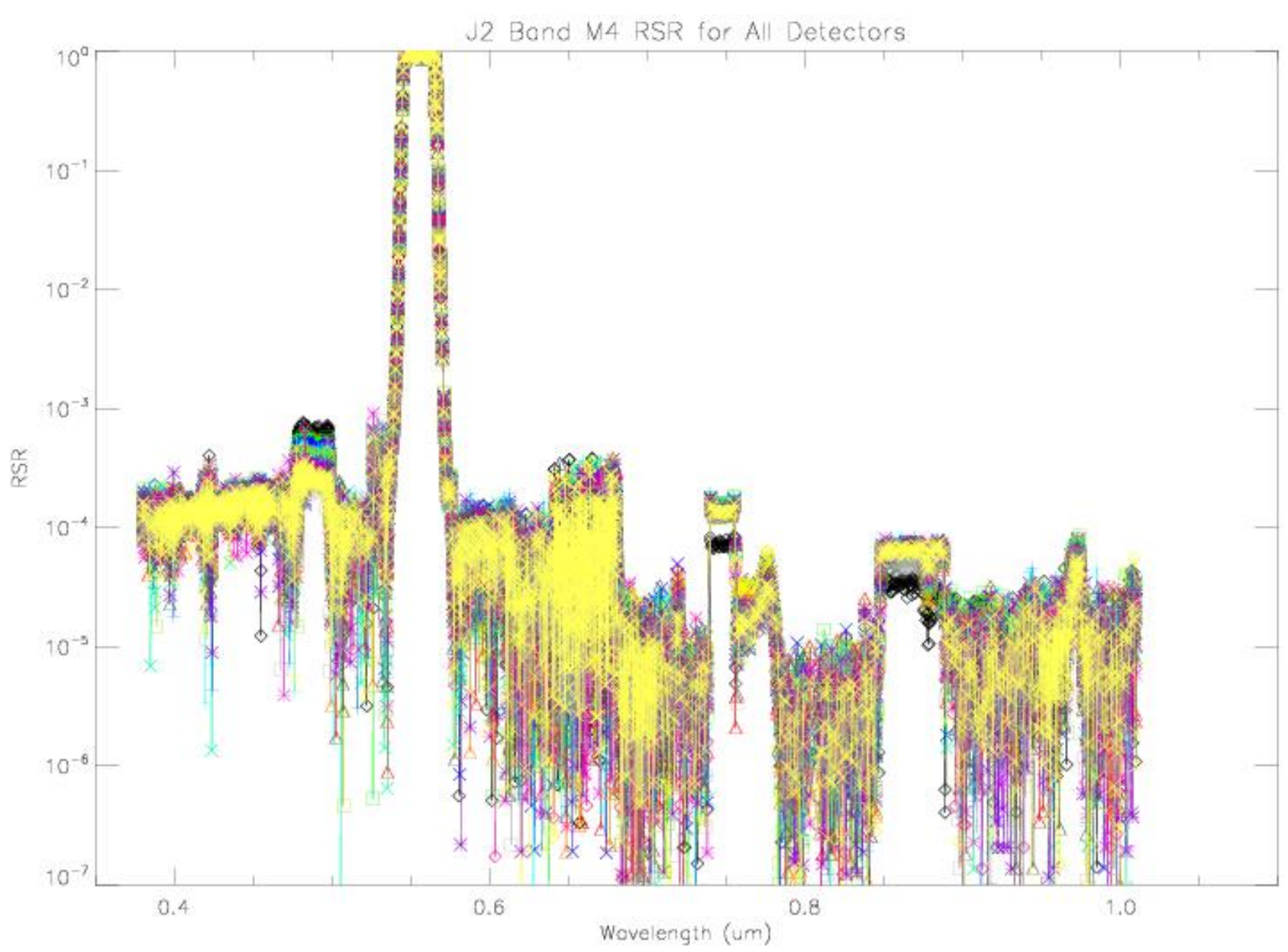

$\diamond 1 \Delta 2 \square 3 \times 4 \quad 5 \times 6 \quad 7 \quad \Delta 8 \quad \square 9 \times 10+11 \quad * 12 \quad \diamond 13 \quad \Delta 14 \quad \square 15 \quad 16$

VIIRS band $\mathrm{M} 2$ showing electronic crosstalk with other bands 


\section{Quasi-CW \& CW sources}

Integrating sphere provides temporal as well as spatial averaging with characteristic time on the order of nanoseconds

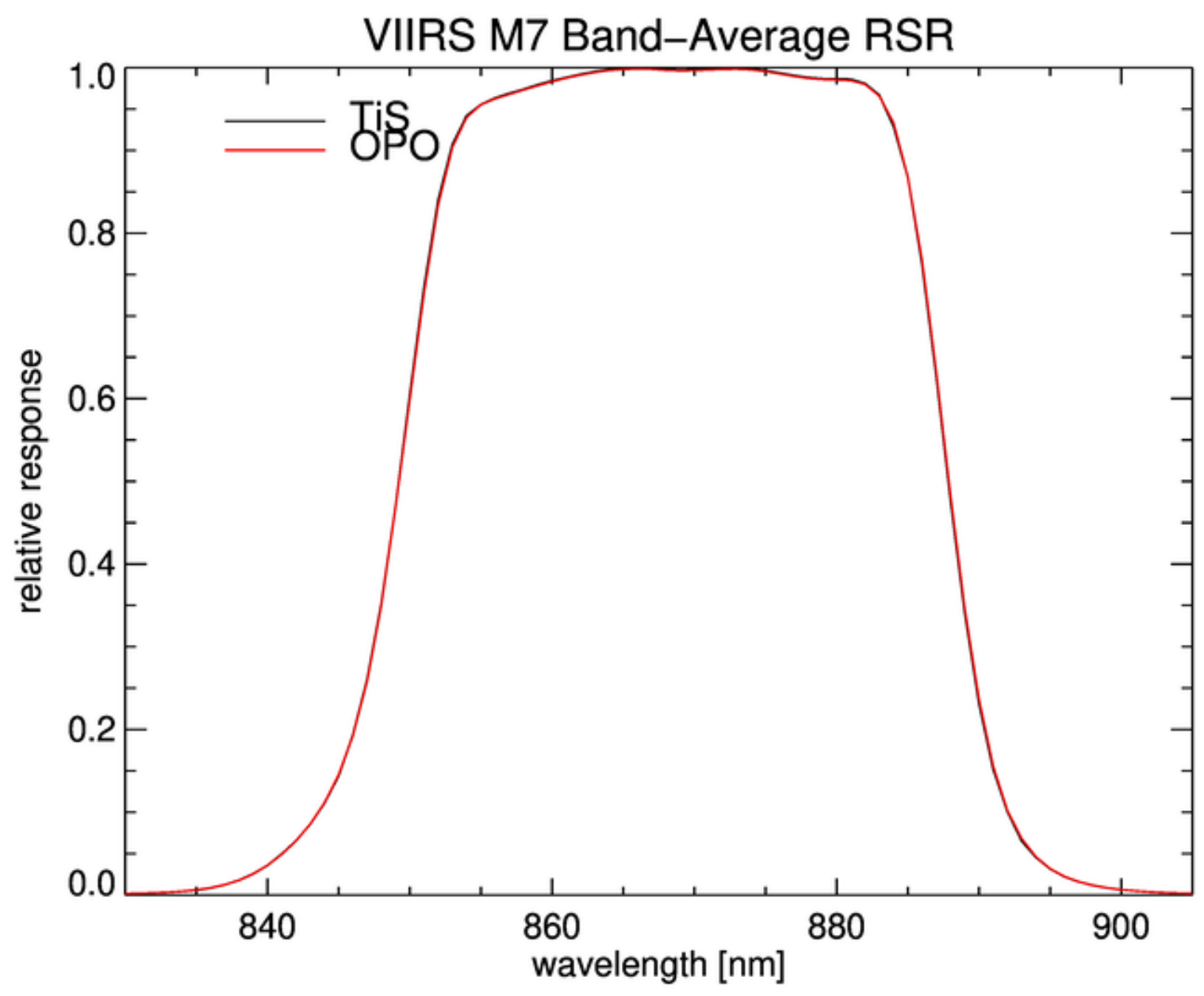

Comparison of $80 \mathrm{MHz}$ prf OPO and continuous wave Ti:sapphire laser used as sources for VIIRS band M7 calibration 


\section{Future work}

- Deeper UV coverage

- Improved operation near degeneracy and water vapor absorption lines

- More repeatable wavelength steps, order of 0.1 $\mathrm{nm}$

- Long term stability and decreased operator intervention during scans

- More power 


\section{Summary, acknowledgements}

Broad band optical parametric oscillator developed covering 360 to $2000 \mathrm{~nm}$

Automated scanning

Demonstrated portability without realignment

Ability to support critical path instrument calibration

Technical Support

Based on design work and built with assistance from the late Keith Lykke, NIST
Support

GOES-R

NPP

SAGE III - ISS

Landsat

PACE Ocean Color Instrument

CLARREO Pathfinder

Joint Polar Satellite System
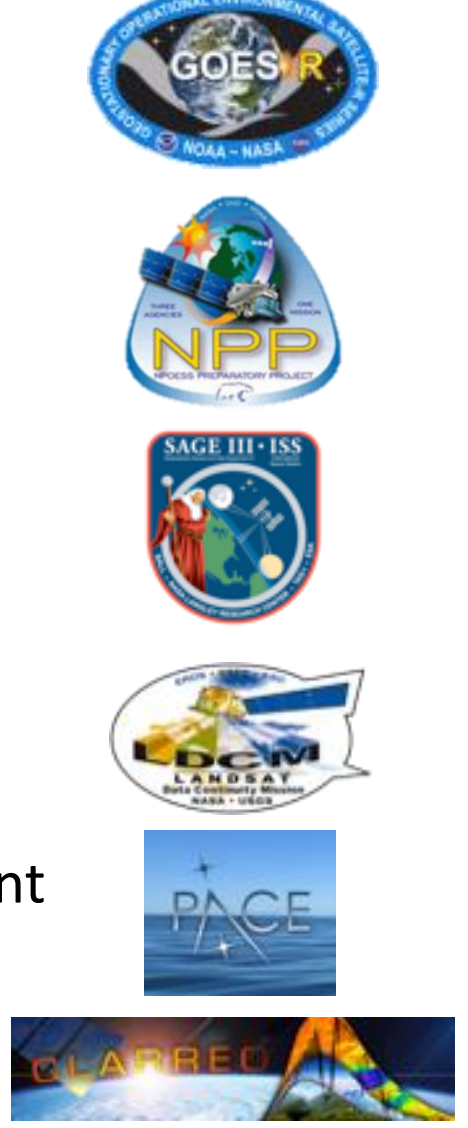

PATHFINDER

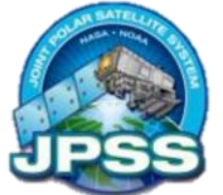

